\title{
Desenvolvimento e simulação de um sistema avançado de controle ambiental em cultivo protegido
}

\author{
Carlos E. B. Romanini ${ }^{1}$, Angel P. Garcia ${ }^{1}$, Leonardo M. Alvarado ${ }^{1}$, N elson L. Cappelli ${ }^{1} \&$ Claudio K. U mezu $^{1}$
}

\section{RESUMO}

A produção em ambientes protegidos é uma técnica por meio da qual se objetiva proteger flores e hortaliças contra a ação dos elementos meteorológicos que a afetam, além de permitir ambientes favoráveis de produção durante todo 0 ano, com vantagens produtivas e comerciais. U m fator importante da produção em estufas é o controle da temperaturae da umidade relativa do ar. A automação do controle do ambiente possibilita o aumento da qualidade dos produtos, maior eficiência e uso racional dos recursos. 0 objetivo do presente trabalho foi desenvolver um sistema de controle de temperatura e umidade relativa do ar para o protótipo de uma ambiente protegido em escala reduzida, utilizando-se dos conceitos da lógica Fuzzy. As variáveis de entrada foram a temperatura e a umidade do ar e as variáveis de saída foram as atuações nos sistemas de aquecimento e de umidificação do ambiente. 0 processo de controle do ambiente foi simulado e os parâmetros do projeto foram ajustados. O s resultados obtidos na simulação demonstraram que os ajustes das variáveis Tar e UR, baseados no sistema de inferência Fuzzy desenvolvido, foram satisfatórios para o desempenho do sistema de controle do ambiente protegido.

Palavraschave: microclima, ambiente protegido, inferência Fuzzy

\section{Development and simulation of an advanced system of ambient control in agricultural greenhouses}

\begin{abstract}
Production in greenhouses is a technique that aims at protecting flowers and vegetable production against meteorological agents that affect production adversely, as well as allowing favorable environments throughout the year with productive and commercial advantages. One important issue regarding greenhouse systems is the control of the air temperature and relative humidity. The automatic environmental control allows the improvement of quality, and the efficient and rational use of resources. The aim of this work was to develop a system of temperature and humidity control for a greenhouse prototype in reduced scale, by using the concepts of Fuzzy Logic. The input variables were: air temperature and humidity, and the output variables were: changes in the environmental heating system and humidification. The environmental control was simulated and project parameters were adjusted. The results of the simulation showed that the air temperature and humidity adjustment based on the inference developed Fuzzy system, was satisfactory for the performance of the greenhouse environmental control.
\end{abstract}

Key words: greenhouse production, environmental control, Fuzzy inference 


\section{INTRODUÇÃO}

A principal diferença entre a produção agropecuária e os demais setores da economia consiste na exposição às condições ambientais. A agricultura é uma atividade de alto risco, uma vez que não se tem controle sobre os elementos climáticos. O conceito de agricultura moderna é produzir de forma sustentável com o menor grau de risco possível, por meio do uso intensivo de conhecimento técnico especializado e tecnologias apropriadas (Assis, 2006). O cultivo realizado em ambientes protegidos é uma das tecnologias que têm contribuído para a modernização da agricultura (Mary et al., 2007).

$\mathrm{O}$ uso do plástico na cobertura de ambiente protegido surgiu como alternativa na proteção de hortaliças e flores, frente às adversidades climáticas (Farias et al., 1993). Mais recentemente, Vida et al. (2004) sintetizaram algumas vantagens do cultivo em ambientes protegidos: aumento de produtividade, colheita na entressafra, precocidade da colheita, maior qualidade dos produtos, melhor controle das condições ambientais, controle mais eficiente de pragas e doenças, melhor aproveitamento no uso dos recursos, minimização do risco e maximização da competitividade mercadológica do produtor. Beltrão et al. (2002) consideram que uma parte significativa da pesquisa agrícola mundial é feita em experimentos sob cultivo protegido.

De acordo com Fontes (1999) e Beltrão et al. (2002), o cultivo em estufa possibilita determinado controle das condições edafoclimáticas, tais como: temperatura, umidade do ar, radiação luminosa, solo, vento e composição atmosférica, e implica na utilização de instrumentação e dispositivos de controle apropriados (Cansado, 2003).

Técnicas não convencionais de controle baseadas na inteligência computacional, como redes neurais artificiais e conjuntos Fuzzy, têm sido crescentemente usadas em sistemas agrícolas (Morimoto et al., 1998). Para Horiuchi \& Kishimoto (2002) os sistemas de controle Fuzzy são eficazes, pois descrevem, em uma rotina, a experiência humana, intuição e heurística para controlar um processo. Existem, na literatura, trabalhos importantes em que se discutem os principais avanços e métodos de controle para cultivos protegidos (Straten et al.,2000; Goggos \& King, 2000; Arvanitis et al., 2000; Setiawan et al., 2000).

No interior do ambiente protegido a temperatura é o fator agrometeorológico que exerce influência sobre as funções vitais das plantas e seu controle é considerado essencial (Purqueiro \& Goto, 2005). Alguns sistemas fazem uso do resfriamento por evaporação da água para redução da temperatura do ar devido à troca do calor sensível por calor latente, o que aumenta a quantidade do vapor de água (Montero et al., 1990). A umidade relativa do ar no interior de um ambiente protegido é influenciada pela temperatura em uma relação inversa entre ambas (Angelocci, 2002). Segundo Andriollo (2000), a UR está vinculada ao equilíbrio hídrico das plantas, em que um déficit pode alterar a evapotranspiração e a capacidade do sistema radicular de absorver água e nutrientes. Pezzopane et al.,(1995) e Furlan \& Folegatti (2002) desenvolveram estudos para caracterizar variações de Tar e UR em ambientes protegidos e observaram que uma variação menor na temperatura do ar ambiente, quando se utilizam sistemas de controle por nebulização.

Diversos estudos recentes exploram alterações nos parâmetros produtivos e na produtividade agrícola, em função do controle de variáveis e modificações microclimáticas em estufa (Galvani et al., 2001; Feltrim et al., 2005; Gusmão et al., 2006; Pires et al., 2006; Trani et al., 2006; Della Vechia et al., 2007; Stidle Neto et al., 2008).

Ante o exposto e se considerando que a temperatura e a umidade afetam o rendimento e a qualidade final de flores e hortaliças objetivou-se, no presente trabalho, desenvolver computacionalmente um sistema de controle no qual se utiliza estratégia de controle avançado para a temperatura e umidade relativa do ar, para um protótipo de casa de vegetação em escala reduzida.

\section{MATERIAL E MÉTODOS}

O presente estudo foi conduzido nas dependências do Laboratório de Instrumentação e Controle (LIC) da Faculdade de Engenharia Agrícola (FEAGRI) da Universidade Estadual de Campinas (UNICAMP), São Paulo, Brasil. Para a realização do ensaio experimental em laboratório, utilizou-se um protótipo em escala reduzida de um ambiente protegido, de acordo com detalhamento metodológico apresentado por Job (2007).

\section{Caracterização do Protótipo}

A estrutura do protótipo foi feita de alumínio, com revestimento externo em plástico transparente para permitir a entrada da luz, de forma análoga às coberturas dos ambientes comerciais protegidos.

Realizou-se o aquecimento do ar no interior do protótipo por meio de duas resistências elétricas de $150 \mathrm{~W}$ cada uma, ligadas em paralelo e acionadas por um relê de estado sólido. A umidificação do ambiente protegido foi realizada com um bico aspersor ligado a uma bomba elétrica automotiva de 12 V. Utilizou-se uma fonte de alimentação com potência de 450 W para o acionamento elétrico dos componentes da estufa.

$\mathrm{O}$ interior do ambiente protegido foi monitorado pela coleta e registro de dados da Tar $\left({ }^{\circ} \mathrm{C}\right)$ e UR (\%), por meio de elementos sensores específicos. A Tar foi mensurada com um sensor LM35 encapsulado enquanto para registrar a UR se utilizou um sensor eletrônico de umidade. A Figura 1 apresenta uma vista frontal do protótipo estudado e o detalhamento da instrumentação utilizada.

\section{Identificação Dinâmica e Modelagem Matemática do Processo}

Visando identificar a dinâmica no protótipo, utilizou-se, no presente trabalho, o método da identificação computacional, que envolveu a coleta de dados das variáveis do processo e a modelagem matemática do comportamento observado experimentalmente. A modelagem teve como objetivo a função de transferência do sistema em estudo, por meio das relações entre a transformada de Laplace da função resposta e a transformada de Laplace da função excitação. 


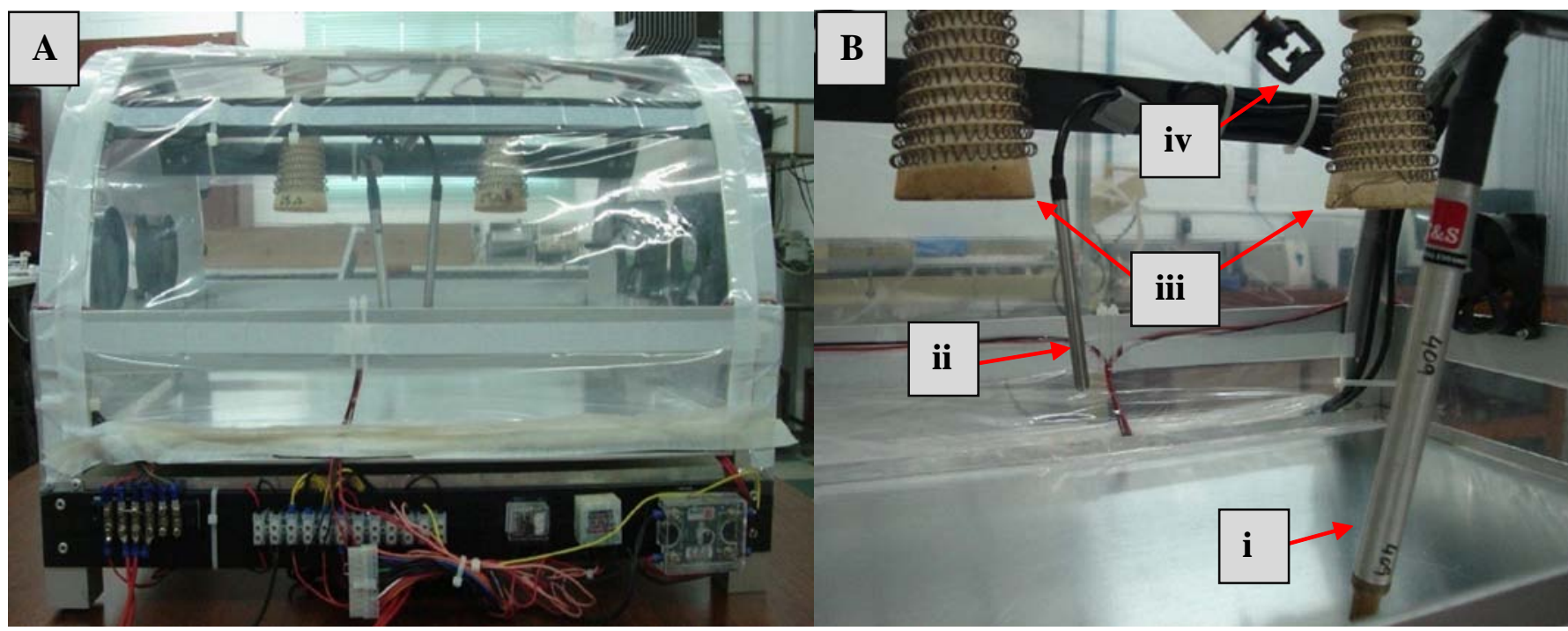

Figura 1. Protótipo em escala reduzida. A. Vista frontal do protótipo; B. Detalhe interno, sendo: i) sensor de Tar; ii) sensor de UR; iii) resistores; iv) bico aspersor de umidificação

Estudou-se a variação dos elementos meteorológicosno protótipo, sem a interferência de um controlador, através da obtenção das respostas temporais das variáveis Tar e UR frente a um estímulo na forma de degrau. O fluxograma apresentado na Figura 2 sintetiza a metodologia experimental aplicada.
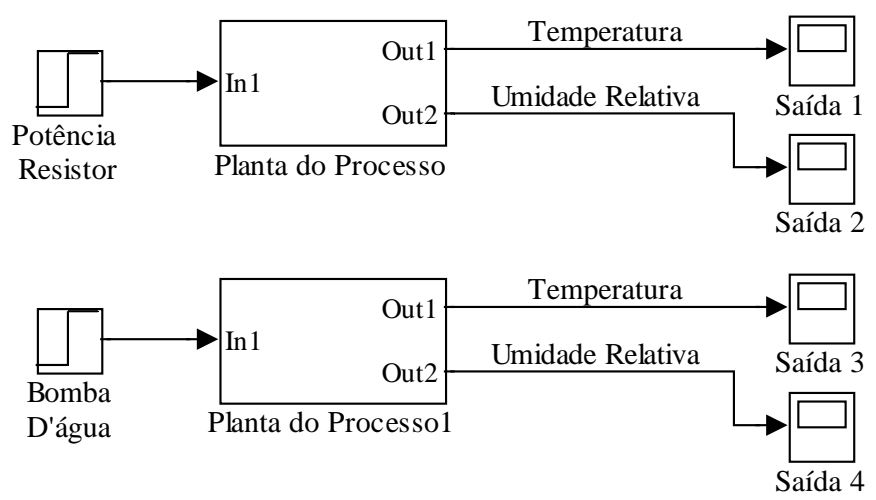

Figura 2. Metodologia para identificar a dinâmica do sistema mediante excitações das variáveis de entrada (elementos atuadores) e reações temporais das variáveis de saída (temperatura e umidade relativa do ar)

A experimentação foi conduzida em duas etapas, na primeira se aplicou um degrau de $50 \%$ de potência na resistência de aquecimento e se verificaram as variações nos valores da Tar (Saída 1) e UR (Saída 2) e na segunda, se aplicou um degrau de $100 \%$ de atuação na aspersão de água, obtendose respostas nas duas variáveis de interesse, Tar (Saída 3) e UR (Saída 4).

Os dados foram aquisitados pelos sensores registrandose, em formato numérico, a interação mútua entre a Tar e a UR, ao longo do tempo. Utilizou-se a representação gráfica do comportamento do sistema para se obter os parâmetros das funções de transferência. A função de transferência característica de um sistema de primeira ordem foi utilizada, com atraso, conforme a Eq. 1 .

$$
G(s)=\frac{K e^{-L s}}{T s+1}
$$

A Eq. 1 é a generalização do modelo que representou uma abstração matemática simplificada do processo real estudado, uma vez que não é possível incorporar todas as características da planta.

\section{Estrutura para simulação do sistema}

Desenvolveu-se uma plataforma de simulação no ambiente Matlab/Simulink. Procedimento conduzido para se obter diferentes respostas temporais das variáveis de interesse, ou seja, Tar e UR, quando da excitação das variáveis de entrada com sinais desejados,por meio da simulação de diversos cenários, para posterior análise. Dentro do ambiente Simulink a linguagem de programação utilizada é gráfica, em forma de blocos. A partir do conhecimento especializado nesta linguagem, desenvolveu-se um diagrama do protótipo, que inclui o uso de um bloco controlador com os conceitos de inferência Fuzzy.

\section{Desenvolvimento do sistema de inferência Fuzzy}

Construiu-se um sistema de inferência Fuzzy no aplicativo específico do Matlab com a mesma estrutura proposta por Mandani (1974) e se desenvolveu um sistema multiváriável Fuzzy composto de quatro variáveis de entrada, sendo: erro da temperatura ar (et), variação do erro da temperatura do ar (vet), erro da umidade relativa do ar (eu) e variação do erro da umidade relativa do ar (veu); e duas variáveis de saída: atuação na temperatura do ar (at) e atuação na umidade relativa do ar (au), conforme ilustra o fluxograma da Figura 3.

\section{RESULTADOS E DISCUSSÃO}

O mecanismo de fuzzificação mapeou os valores reais obtidos dos sensores das variáveis de entrada e os transformou em variáveis linguísticas definidas por funções de per- 


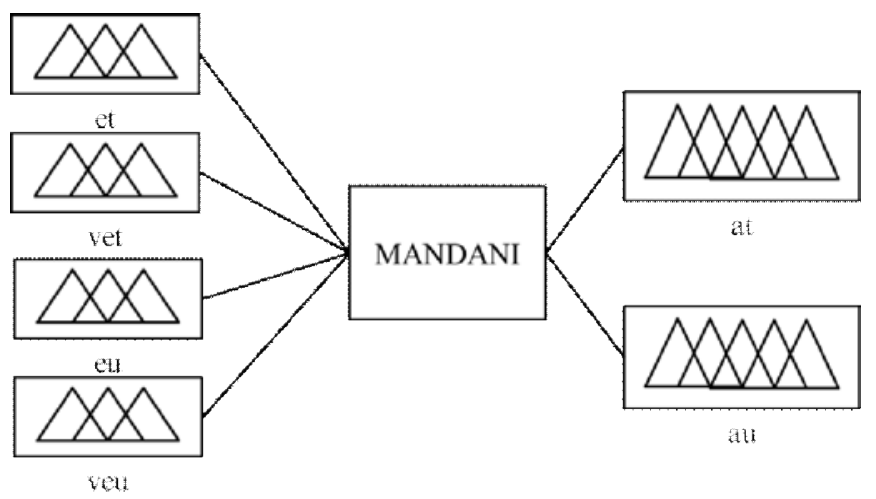

Figura 3. Fluxograma do sistema de controle fuzzy

tinência triangulares com cardinalidade igual a 3 (três), já que, em processos multivariáveis, uma das maiores dificuldades no projeto está relacionado à dimensão e ao tamanho da base de regras, que muitas vezes pode tornar o controle impraticável para aplicações em tempo real. Na Figura 4 se apresentam as variáveis linguísticas e suas respectivas funções de pertinência.

De modo similar, o mecanismo de fuzzyficação fez decodificação da atuação no sistema. Transformaram-se as variáveis Fuzzy produzidas pela inferência em uma variável numérica, que se interligou ao processo por meio dos elementos atuadores, de forma a regulá-lo. Uma particularidade para as variáveis de saída (at, au) do sistema Fuzzy foi o uso de cardinalidade igual a cinco, para as funções de pertinência também triangulares, conforme ilustra a Figura 5.

Desenvolveu-se um conjunto composto de 81 regras denominadas produção Fuzzy, formadas por duas partes principais, sendo: if < antecedente > then < conseqüente >. O elemento antecedente da regra foi formado por um conjunto de condições das variáveis de entrada as quais, quando satisfeitas, determinaram o processamento do elemento consequente da regra, que consistiu de um conjunto de ações estabelecidas para as variáveis de saída do sistema. A superfície de resposta de atuação em função das variáveis de entrada é apresentada nos gráficos da Figura 6.

Os resultados obtidos do comportamento das variáveis de interesse (Tar e UR), conforme metodologia da Figura 2, em função do tempo, sem um elemento controlador, estão apresentados na Figura 7.

O método da resposta ao degrau se baseou no conhecimento de algumas grandezas que caracterizaram a resposta dinâmica do processo, de acordo com procedimento clássico disponível na literatura de engenharia de controle e automação (Ogata, 2003; Bazanella \& Silva Jr., 2005). Com isto, obtiveram-se os valores da constante de proporcionalidade $(\mathrm{K})$, que é o ganho estático do processo, da constante de tempo (T) e da constante (L), correspondente ao atraso do sistema. Os valores das constantes para cada caso estudado estão apresentados na Tabela 1.

Com a finalidade de se desenvolver um controlador para a estufa estudada com requisitos apropriados, defendidos por Cansado (2003), o comportamento dinâmico do processo resultou em diferentes modelos matemáticos, conforme a Eq. 1 e a partir dos valores das constantes apresentados na Tabela 1. Utilizando-se do conhecimento extraído dos modelos, de-

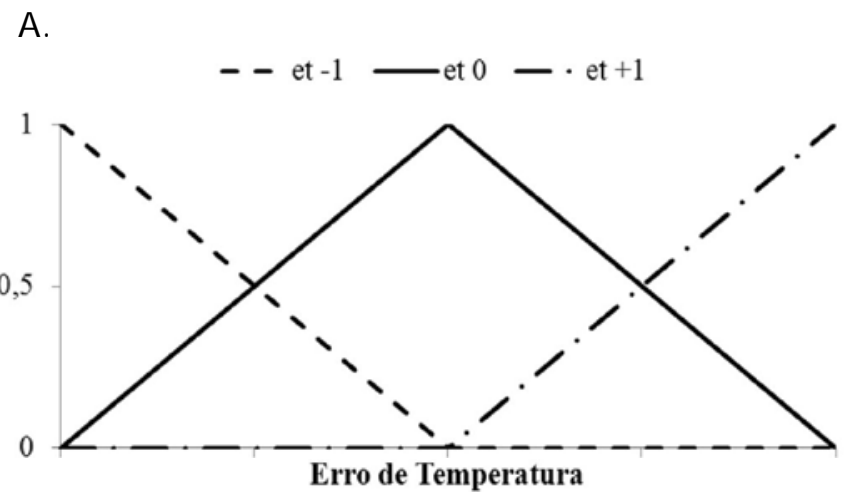

B.

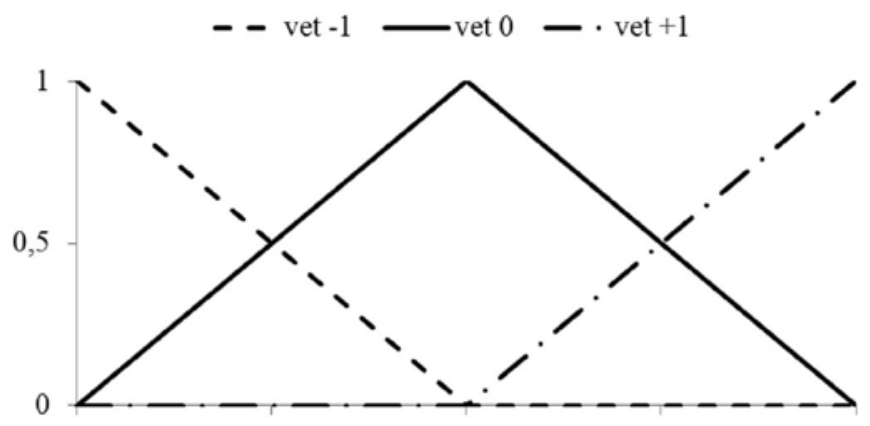

Variação do Erro de Temperatura

C.

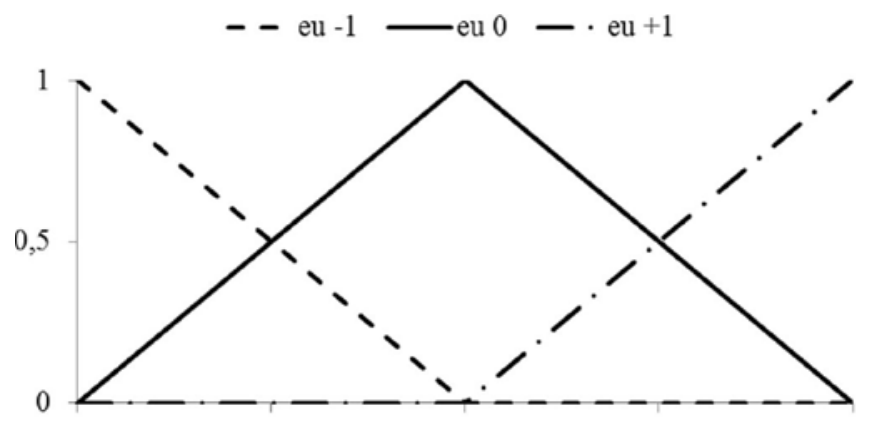

Erro da Umidade Relativa

D.

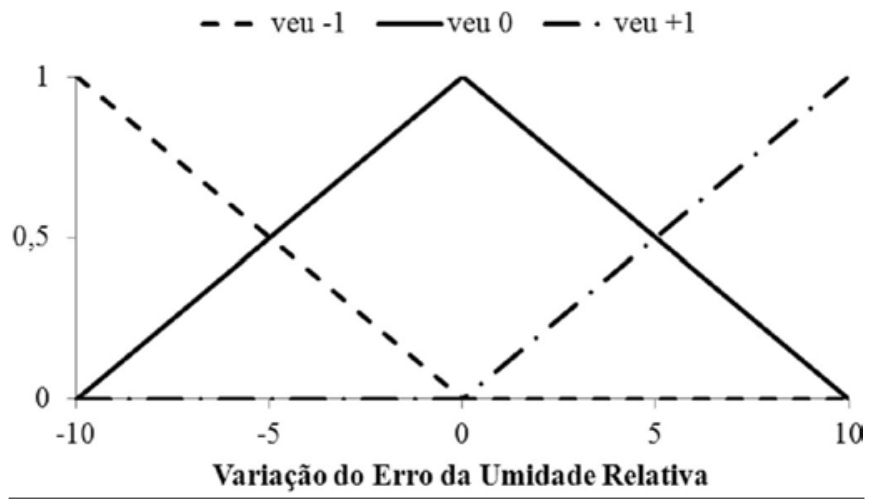

Figura 4. Funções de pertinência das variáveis de entrada do sistema de controle fuzzy. A. erro de temperatura; $B$. variação do erro de temperatura; $C$. erro de umidade relativa; $D$. variação do erro da umidade relativa

terminou-se a capacidade de influência no ajuste da temperatura e umidade relativa do ar dentro da estufa, conforme necessidades particulares do clima da região produtora, do produtor ou tipo de cultivo, consistente com as afirmações apresentadas por Fontes (1999) e Beltrão et al. (2002). 
Tabela 1. Valores das constantes identificadas dos sistemas, obtidas experimentalmente de acordo com o caso estudado (conforme Figura 2)

\begin{tabular}{crccc}
\hline \multirow{2}{*}{ Caso Estudado } & \multicolumn{3}{c}{ Valores das constantes } \\
\cline { 3 - 5 } \multicolumn{2}{c}{ Caso 1 } & K & $\mathbf{T}$ & $\mathrm{L}$ \\
& Saída 1 & 31,32 & 875 & 225 \\
& Saída 2 & $-46,44$ & 750 & 200 \\
\hline \multirow{2}{*}{ Caso 2 } & Saída 3 & $-1,26$ & 250 & 40 \\
& Saída 4 & 49,21 & 55 & 1 \\
\hline
\end{tabular}

A.

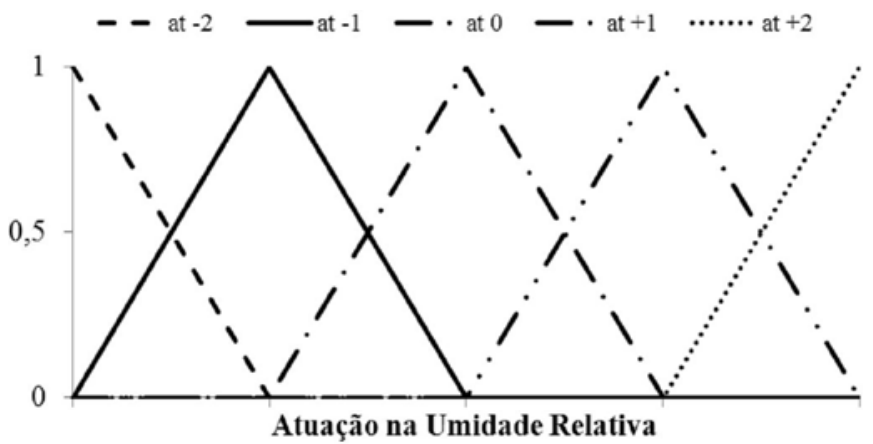

B.

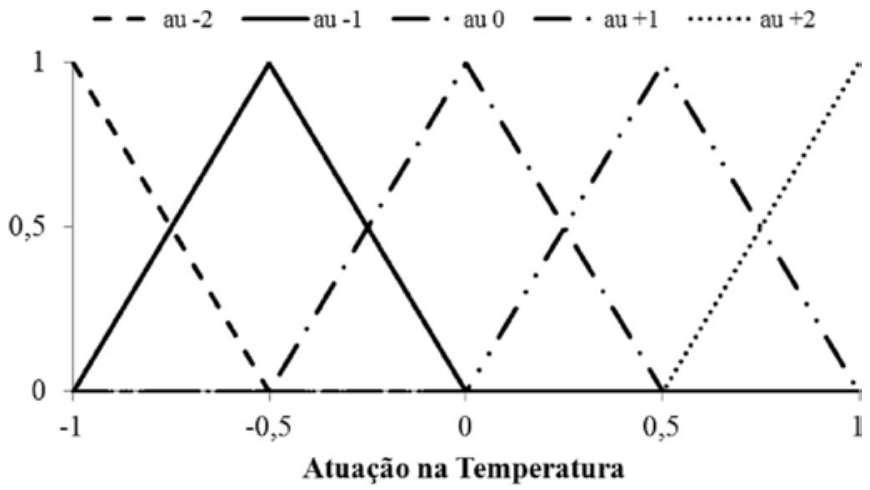

Figura 5. Funções de pertinência das variáveis de saída do sistema de controle fuzzy. A. atuação na temperatura; B. atuação na umidade relativa
Os modelos foram utilizados em simulações no ambiente MatLab/Simulink, cujos diagramas de bloco estão apresentados nas Figuras 8 e 9.

Três cenários de excitação na entrada do processo foram simulados como exemplo e verificadas as respostas temporais gráficas das variáveis de saída, temperatura e umidade relativa do ar. Os gráficos da Figura 10 apresentam, respectivamente, os resultados das simulações com tempo de 3500 segundos para 3 (três) diferentes perturbações de incremento de set point, sendo: $5{ }^{\circ} \mathrm{C}$ de temperatura do ar; $5 \%$ de umidade relativa do ar; $5{ }^{\circ} \mathrm{C}$ de temperatura e $5 \%$ de umidade relativa simultaneamente.

Os principais fatores de desempenho das respostas do sistema obtidos na simulação estão descritos a seguir. No regime transitório e se considerando todas as excitações efetuadas, obteve-se um máximo sobressinal de $12 \%$ para a variável umidade relativa do ar e de $4 \%$ para a temperatura do ar. Como os valores de sobressinal obtidos são relativamente pequenos, o sistema é caracterizado por um comportamento estável. Em algumas situações agrícolas o sobressinal é altamente indesejável visto que inviabiliza o desenvolvimento de animais e plantas. Para o caso das estufas agrícolas e se considerando que a temperatura é o fator essencial para o desenvolvimento das plantas e que a umidade atua de forma secundária, os valores obtidos estão consistentes com exigências apresentadas na literatura (Angelocci, 2002; Purqueiro \& Goto, 2005).

O tempo de subida das variáveis respostas obtidos foram um indicativo de quão rápido o sistema reage à aplicação de uma entrada, caso em que o tempo que decorrido para que a resposta fosse de 10 a $90 \%$ do valor final, foi de aproximadamente $30 \mathrm{~s}$ para a umidade relativa do ar e de $250 \mathrm{~s}$ para a temperatura. Com os referidos valores é possível explicar o maior sobressinal da variável umidade relativa do ar. O tempo de acomodação para a simulação de perturbações foi de 250 s para a umidade relativa do ar e de $1500 \mathrm{~s}$ para a temperatura do ar; notou-se, desta forma, que modificações microclimáticas ocorreram na estufa, porém a função do controlador foi

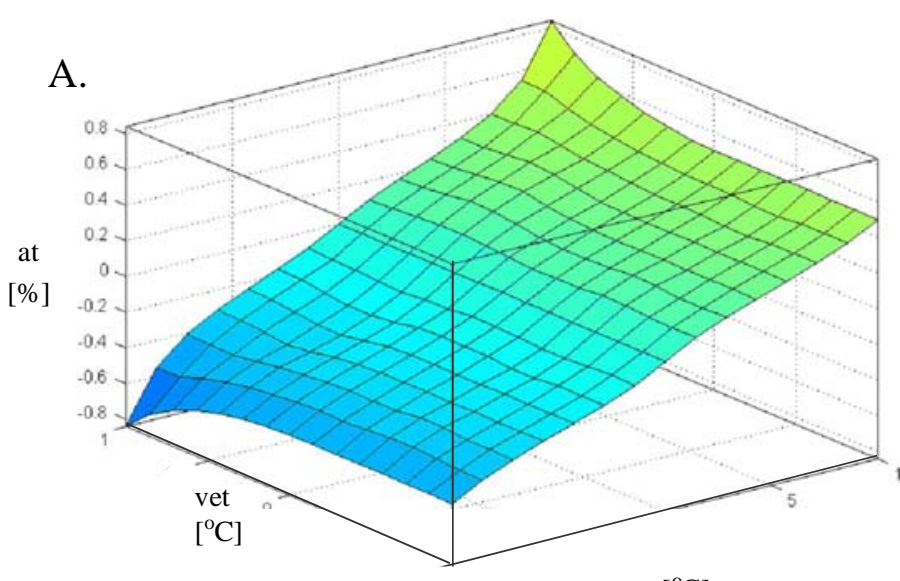

et $\left[{ }^{\circ} \mathrm{C}\right]$

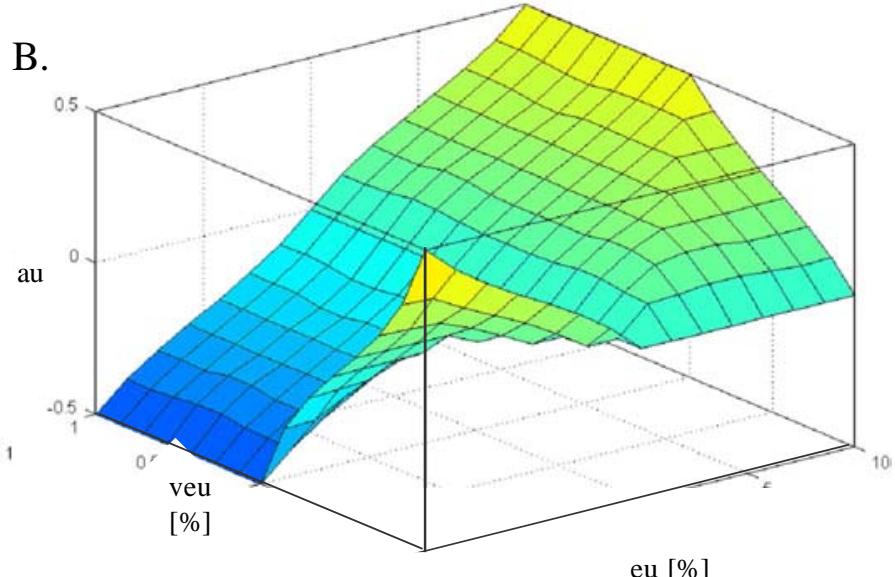

$\mathrm{eu}[\%]$

Figura 6. Superfície de resposta das principais regras definidas. A.Atuação na temperatura em função do erro de temperatura e variação do erro de temperatura; B.Atuação na umidade relativa em função do erro da umidade relativa e variação do erro da umidade relativa 
A.
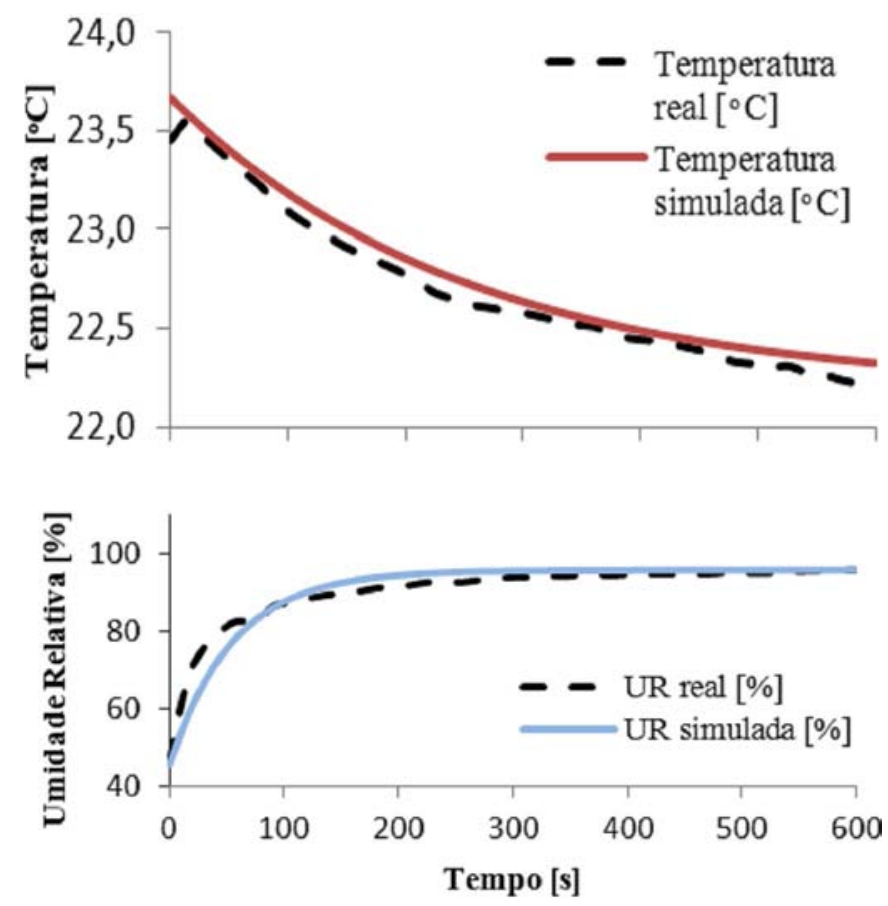

B.
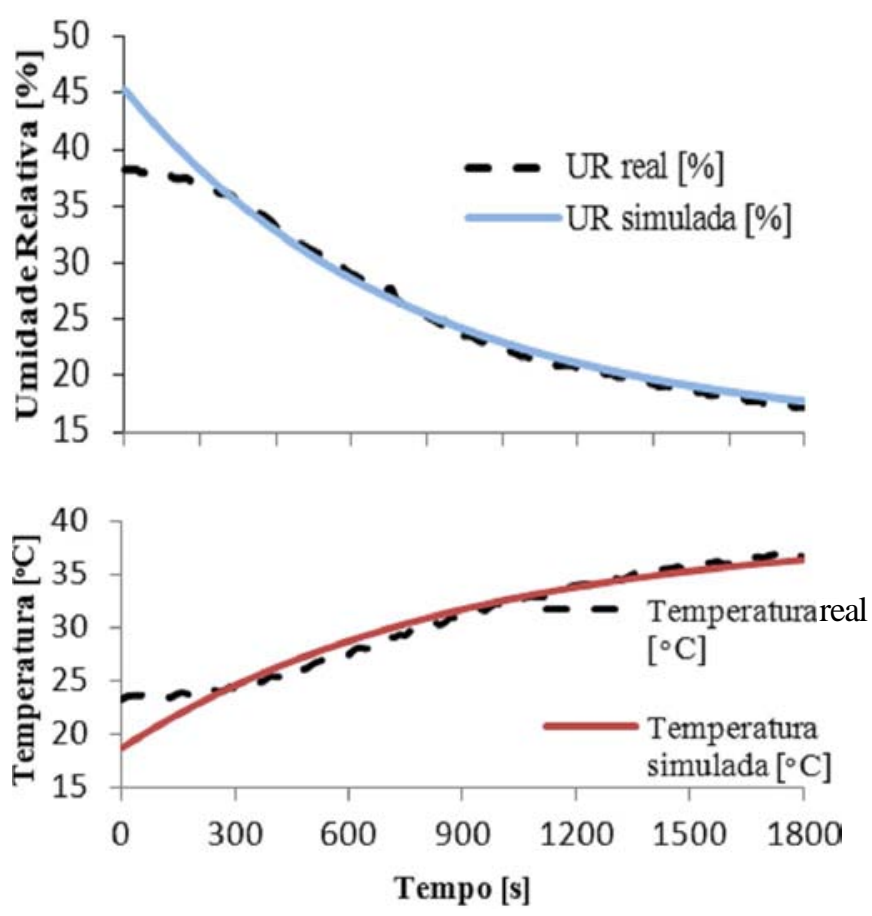

Figura 7. Respostas das variáveis estudadas (temperatura e umidade relativa) frente a uma excitação do tipo degrau. A. 50\% na potência das resistências de aquecimento. B.100\% de potência na bomba de aspersão deágua

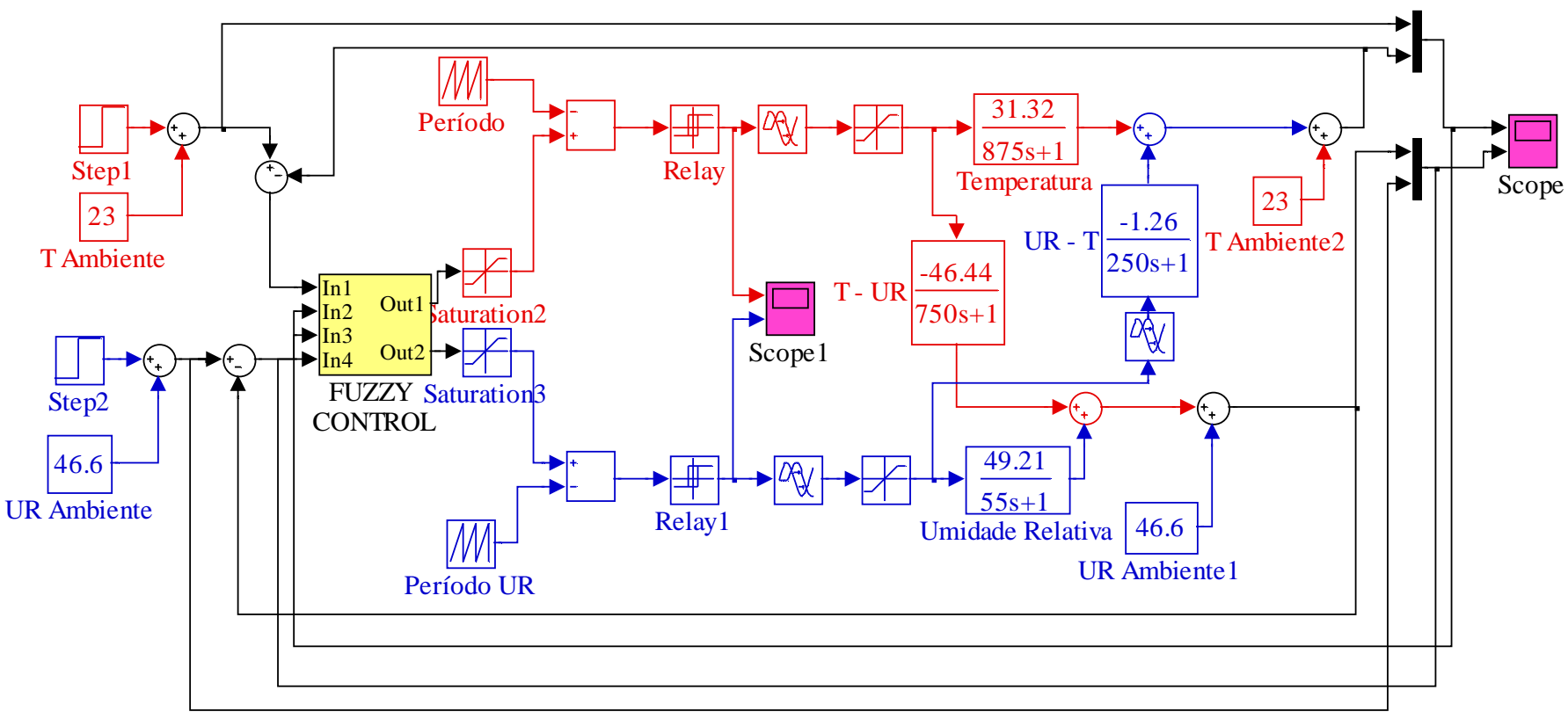

Figura 8. Diagrama de blocos do programa desenvolvido em ambiente MatLab/Simulink para simulação do protótipo da estufa

efetuada com êxito, pois permitiu atingir, em tempo curto, o ambiente desejado, apesar das flutuações ocorridas; resultados semelhantes foram discutidos por Straten et al. (2000). A uniformização do ambiente da estufa, caracterizado por diversos pontos de registro de dados, deve ser considerada para o desempenho do controlador em situações reais práticas (Beltrão et al., 2002; Furlan \& Folegatti, 2002).

$\mathrm{O}$ atraso de transporte na variável temperatura do ar foi de aproximadamente $250 \mathrm{~s}$, evidenciando uma inércia térmica já prevista. Este é o tempo necessário para que a variação no sinal de entrada seja observada na temperatura; por sua vez, tal atraso é uma somatória dos atrasos provocados pelo sensor, operação do elemento atuador e ação do próprio controlador; em geral, quanto maior o atraso mais difícil é o controle do processo.

Observou-se, a partir das simulações executadas, que as configurações das regras Fuzzy estabelecidas com o auxílio de um especialista, foram capazes de resolver alguns confli- 


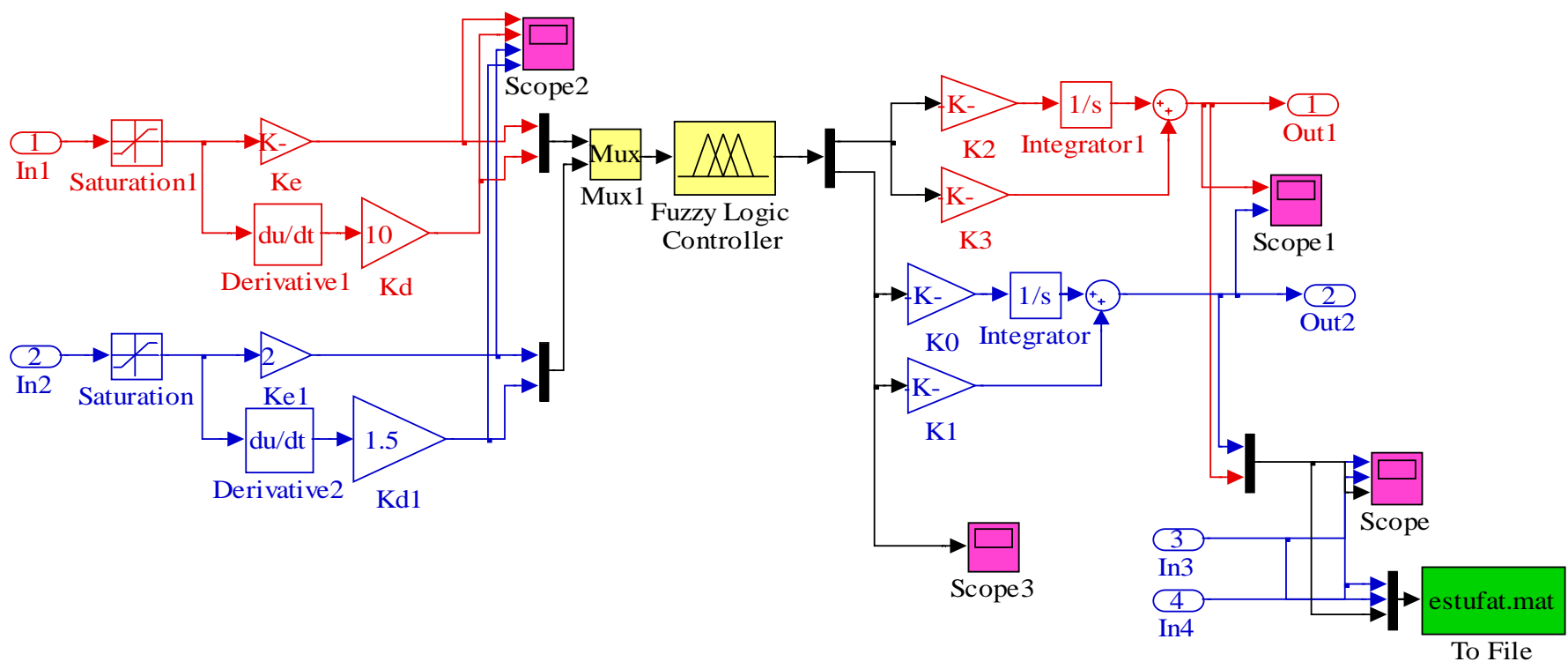

Figura 9. Subsistema de controle Fuzzy desenvolvido em ambiente M atLab/Simulink para simulação do protótipo da estufa

A1.

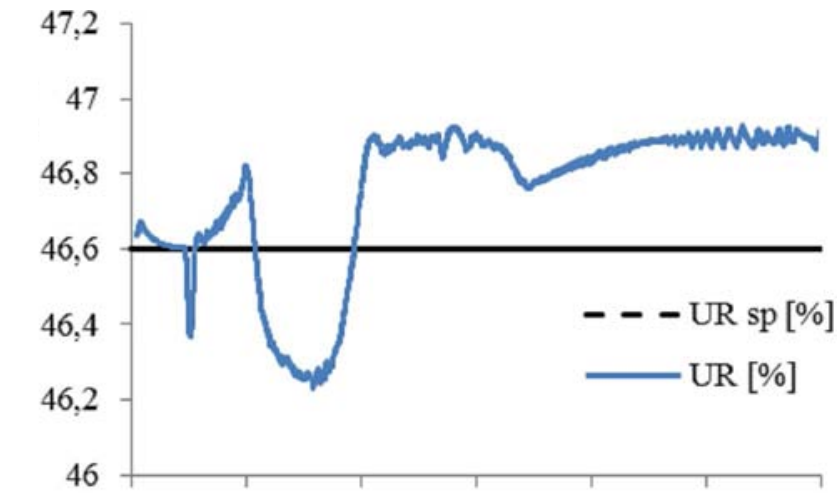

B1.

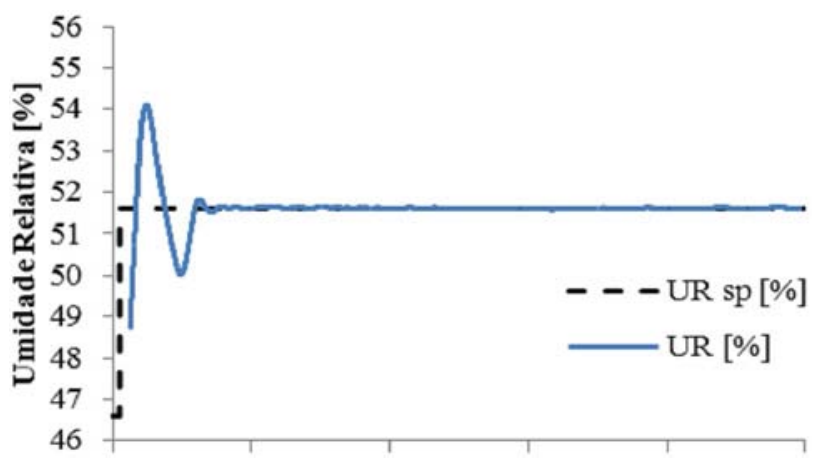

C1.

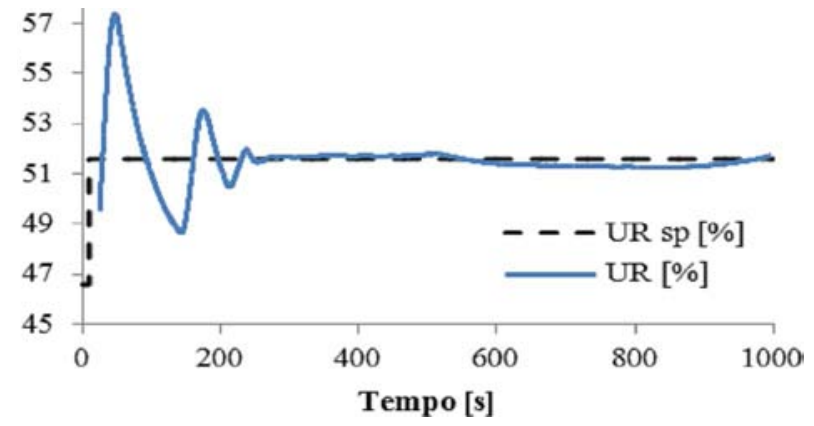

A2.

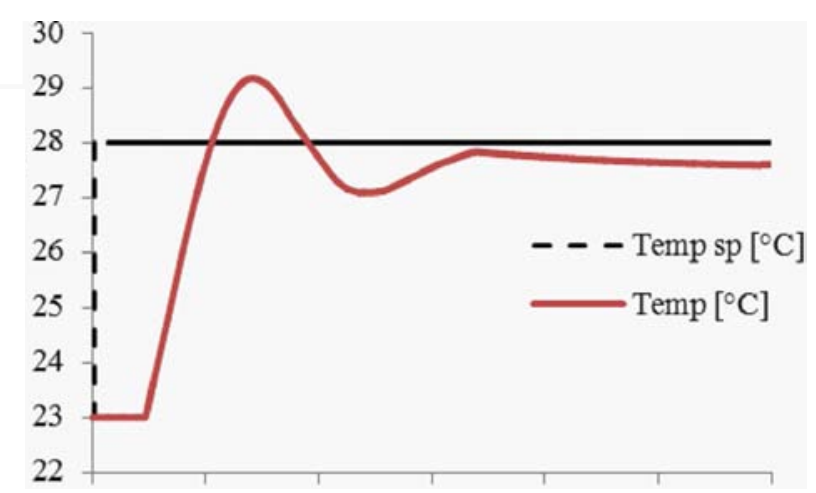

B2.

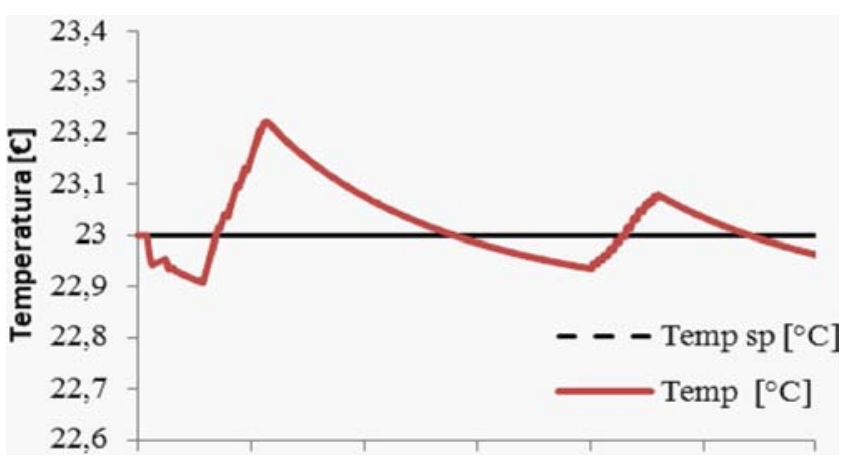

C2.

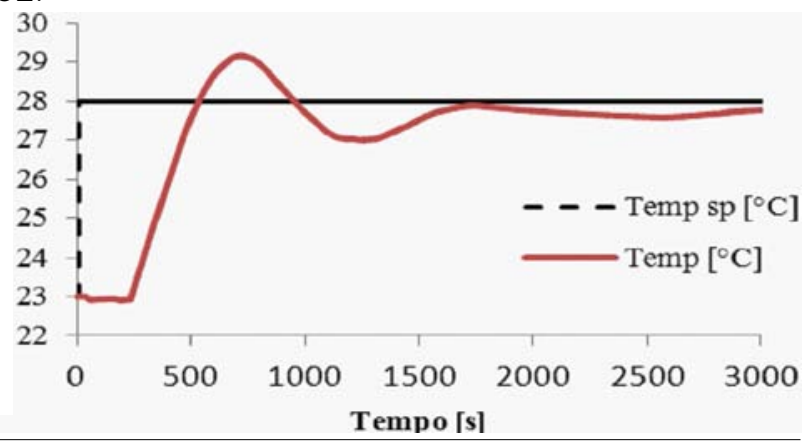

Figura 10. Resposta da umidade relativa (\%) e da temperatura do ar (드) diante da variação do setpoint A. variação de $5 \%$ no setpoint da umidade relativa; B. variação de 5 ㅇ $\mathrm{C}$ no set point da temperatura do ar; C.variação simultânea do set point de 5 으 na temperatura e $5 \%$ na umidade relativa 
tos existentes entre a atuação do controlador de temperatura e a umidade; este resultado coincide com as conclusões de van Stranten et al. (2000) de que os algoritmos mais modernos de controle devem ser concebidos de maneira mais heurística e considerando a interação entre os fatores que influenciam o processo; por exemplo; temperatura, umidade e concentração de dióxido de carbono, de forma não linear mas que condicione a atuação dos sistemas de aquecimento ou resfriamento.

O desempenho apresentado expressa os objetivos alcançados na simulação, com a execução da sintonia e ajuste adequado dos parâmetros do sistema de controle com lógica Fuzzy, sobretudo na adequação do conjunto de regras; com a simulação foi possível analisar, com certa precisão, o comportamento das variáveis para qualquer condição de operação concordando, assim, com as considerações de Straten et al. (2000) que consideram o uso da simulação e o controle computacional como parte fundamental do desenvolvimento de sistemas modernos de produção agrícola em estufas.

O sistema computacional de controle desenvolvido permite ao produtor ajustar as configurações e as trajetórias desejadas de temperatura e umidade relativa do ar, de acordo com as observações da performance da cultura, com base na sua experiência e no conhecimento.

A tendência para sistemas de controle modernos em estufa é discutida na literatura. Arvanitis et al. (2000) afirmam que sistemas de controle semelhantes ao desenvolvido no presente trabalho, que fazem uso de técnicas baseadas na inteligência computacional, como é a Lógica Fuzzy, estão migrando com sucesso das aplicações industriais para a agricultura. O sistema de controle computacional aqui desenvolvido é consistente com as expectativas enunciadas por Goggos \& King (2000), pois se constitui no passo inicial para o desenvolvimento de sistemas hierárquicos de controle híbrido, com a fusão de técnicas de inteligência computacional e computação evolutiva.

Conforme conclusões de Goggos \& King (2000), altas produtividades e qualidade produtiva somente são alcançadas nas estufas agrícolas se obedecidas as condições ideais de ambiente e de nutrientes para a planta. Entende-se que o controlador desenvolvido cumpre as necessidades de um ambiente ideal na estufa e sua implementação real pode resultar em benefícios produtivos e econômicos, recentemente apresentados por Trani et al. (2006), Della Vechia et al. (2007) e Stidle Neto et al. (2008).

\section{CONCLUSÕES}

1. Os resultados obtidos e apresentados no presente estudo demonstraram que o controle simultâneo das variáveis temperatura e umidade relativa do ar, baseado em um sistema de inferência Fuzzy, foi satisfatório para simulação do desempenho do sistema de controle do ambiente para a estufa estudada em um protótipo em escala reduzida.

\section{LITERATURA CITADA}

Andriollo, J. L. Fisiologia da produção de hortaliças em ambiente protegido. Horticultura Brasileira, v.18, n.1, p.26-33, 2000.

Angelocci, L. R. Água na planta e trocas gasosas/energéticas com a atmosfera: Introdução ao tratamento biofísico. 1.ed. Piracicaba: Autor, 2002. 272p.

Arvanitis, K. G.; Paraskevopoulos, P. N.; Vernardos, A. A. Multirate adaptive temperature control of greenhouses. Computers and Electronics in Agriculture, v.26, n.3, p.303-320, 2000.

Assis, R. L. Desenvolvimento rural sustentável no Brasil: perspectivas a partir da integração de ações públicas e privadas com base na agroecologia. Economia Aplicada, v.10, n.1, p.7589, 2006.

Bazanella, A. S.; Silva Jr., J. M. G. Sistemas de controle: Princípios e métodos de projeto. 1.ed. Porto Alegre: UFRGS, 2005. 292p.

Beltrão, N. E. de M.; Fideles Filho, J.; Figeuirêdo, I. C. M. Uso adequado de casa-de-vegetação e de telados na experimentação agrícola. Revista Brasileira de Engenharia Agrícola e Ambiental, v.6, n.3, p.547-552, 2002.

Cansado, J. C. A. Agrilogic: Sistema para experimentação de controle de casas de vegetação. Escola Politécnica/Universidade de São Paulo. 2003. 118p. Dissertação Mestrado

Della Vecchia, M. G. S.; Rosa, D. D.; Bergamin Filho, A.; Amorim, L.; Rezende, J. A. M.; Ribeiro, A. Dinâmica temporal e espacial da begomovirose causada por Tomato yellow vein streak vírus em tomateiro na região de Campinas-SP. Summa Phytopathologica, v.33, n.4, p.388-396, 2007.

Farias, J. R. B.; Bergamaschi, H.; Martins, S.R.; Berlato, M. A.; Oliveira, A. C. B. Alterações na temperatura e umidade relativa do ar provocado pelo uso de estufa plástica. Revista Brasileira de Agrometeorologia, v.1, n.1, p.51-62, 1993.

Feltrim, A. L.; Cecílio Filho, A. B.; Branco, R. B. F.; Barbosa, J. C.; Salatiel, L. T. Produção de alface americana em solo e em hidroponia, no inverno e verão, em Jaboticabal, SP. Revista Brasileira de Engenharia Agrícola e Ambiental, v.9, n.4, p.505-509, 2005.

Fontes, P. C. R. Produção de hortaliças em ambiente protegido: uma técnica a ser aprendida. Informe Agropecuário, v.20, n.200/201, p.1-2, 1999.

Furlan, R. A.; Folegatti, M. V. Distribuição vertical e horizontal de temperaturas do ar em ambientes protegidos. Revista Brasileira de Engenharia Agrícola e Ambiental, v.6, n.1, p.93-100, 2002.

Galvani, E.; Escobedo, J. F.; Pereira, A. B. Balanço de radiação e fluxo de calor no solo em ambiente natural e protegido cultivado com pepineiro. Bragantia, v.60, n.2, p.139-147, 2001.

Goggos, V.; King, R. E. Qualitative-evolutionary design of greenhouse environment control agents. Computers and Electronics in Agriculture, v.26, n.3, p.271-282, 2000.

Gusmão, M. T. A; Gusmão, S. A. L.; Araújo, J. A. C. Produtividade de tomate tipo cereja cultivado em ambiente protegido e em diferentes substratos. Horticultura Brasileira, v.24, n.4, p.431-436, 2006.

Horiuchi, J.; Kishimoto, M. Application of Fuzzy control to industrial bioprocess in Japan. Fuzzy sets and Systems, v.128, n.1, p.117-124, 2002. 
Job, E. E. Protótipo experimental de uma estufa com controle automatizado de temperatura e umidade. Campinas: Faculdade de Engenharia Mecânica/ Universidade São Francisco, 2007. 41p.

Mary, W.; Kenmochi, C. S.; Cometti, M. N.; Leal, P. A. M. Avaliação de estrutura de bambu como elemento construtivo para casa de vegetação. Engenharia Agrícola, v. 27, n.1, p.100-109, 2007.

Mamdani E. H., Applications of Fuzzy algorithms for control of a simple dynamic plant. Proc. IEE, v.121, p.1585-1588, 1974.

Montero, J. I.; Antón, A.; Biel, C.; Franquet, A. Cooling of greenhouse with compressed air fogging nozzles. Acta Horticulturae, v.281, n.1, p.199-209, 1990.

Morimoto T.; Hashimoto Y.; Hoshi T. An intelligent control technique based on Fuzzy controls, neural networks and genetic algorithms for greenhouse automation. In: IFAC-CIGR Workshop, 3, 1998, Artificial intelligence in agriculture. Proceedings ... Chiba: Pergamon/Elsevier Science. 1998. p.61-66.

Ogata, K. Engenharia de controle moderno. 4.ed. São Paulo: Pearson Prentice Hall, 2003. 788p.

Pezzopane, J. E. M.; Pedro Júnior, M. J.; Ortolani, A. A. Modificações microclimáticas provocadas por estufas com cobertura plástica. Bragantia, v.54, n.2, p.419-425, 1995.

Pires, R. C. M.; Folegatti, M. V.; Passos, F. A.; Arruda, F. B.; Sakai, E. Vegetative growth and yield of strawberry under irrigation and soil mulches for different cultivation environments. Scientia Agrícola, v.63, n.5, p.417-425, 2006.
Purquerio, L. F. V.; Goto, R. Dose de nitrogênio em cobertura via fertirrigação e espaçamento entre plantas sobre a cultura da rúcula, em campo e ambiente protegido. In: Congresso Ibérico de Ciências Hortícolas, 5, e Congresso Iberoamericano de Ciências Hortícolas, 4, 2005, Porto. Anais ... Porto: Actas Portuguesas de Horticultura, 2005. p.336-341.

Setiawan, A.; Albright, L. D.; Phelan, R. M. Application of pseudo-derivative-feedback algorithm in greenhouse air temperature control. Computers and Electronics in Agriculture, v.26, n.3, p.303-320, 2000.

Steidle Neto, A. J.; Zolnier, S.; Marouelli, W. A.; Carrijo, O. A. Razão entre radiação fotossinteticamente ativa e radiação global no cultivo do tomateiro em casa-de-vegetação. Revista Brasileira de Engenharia Agrícola e Ambiental, v.12, n.6, p.626-631, 2008.

Straten, G. van; Challa, H.; Buwalda, F. Towards user accepted optimal control of greenhouse climate. Computers and Electronics in Agriculture, v.26, n.3, p.221-238, 2000.

Trani, P. E.; Novo, M. C. S. S.; Cavallaro Júnior, M. L.; Gonçalves, C.; Maggio, M. A.; Giusto, A. B.; Vailati, M. L. Desempenho de cultivares de alface sob cultivo protegido. Bragantia, v.65, n.3, p.441-445, 2006.

Vida, J. B.; Zambolim, L.; Tessmann, D. J.; Brandão Filho, J. U. T. Jaqueline R.Verzignassi, J. R.; Caixeta, M. P. Manejo de doenças de plantas em cultivo protegido. Fitopatologia Brasileira, v.29, n.4, p.355-372, 2004. 